\title{
Sporadic inclusion body myositis: clinical, pathological, and genetic analysis of eight Polish patients
}

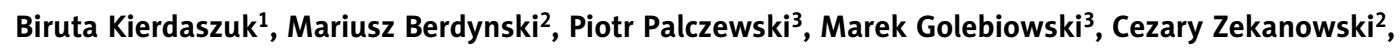 \\ Anna Maria Kaminska ${ }^{1}$ \\ ${ }^{1}$ Department of Neurology, Medical University of Warsaw, ${ }^{2}$ Department of Neurodegenerative Disorders and Neurogenetic Unit, \\ Mossakowski Medical Research Center, Polish Academy of Sciences, Warsaw, ${ }^{3} 1^{\text {st }}$ Department of Clinical Radiology, Medical University \\ of Warsaw, Poland
}

\begin{abstract}
Introduction: Sporadic inclusion body myositis (SIBM) is one of the most common myopathies in patients above 50 years of age. Its progressive course finally leads to immobilisation, and no effective therapy exists. Its pathogenesis includes both degenerative and inflammatory processes, however, its direct causes remain unknown. Therefore, a possible genetic background of the disease must also be considered.

Material and methods: Here we report on twelve patients: eight with sporadic inclusion body myositis and four with other myopathies with rimmed vacuoles in muscle biopsy. All patients were evaluated clinically, morphologically, radiologically, and genetically.

Results: All patients with sIBM presented both shoulder and pelvic girdle muscle involvement. In addition, distal upper and lower limb muscle weakness was noted. Patients with other muscle disorders showed effects mainly in proximal muscles and marked calf muscle hypertrophy. In sIBM cases computed tomography of lower limb muscles revealed atrophy that was most pronounced within the quadriceps femoris and gracilis muscles in the thighs and within the medial head of the gastrocnemius muscle and the tibialis anterior muscle in the lower legs. On light microscopy mononuclear cell invasion of muscle fibres was present in six patients with sIBM. On electron microscopy myofibrillar disorganisation and mitochondrial abnormalities were noted in all SIBM patients, whereas cytoplasmic tubulofilamentous inclusions were seen in three patients and both cytoplasmic and nuclear inclusions in one of them. According to the criteria by Rose et al. (2011) six patients were classified as "clinico-pathologically defined IBM", one as "clinically defined IBM", and one as "probable IBM". Pathological deposits of TDP-43 were found in muscles in all SIBM as well as in control cases. Additionally, accumulation of other proteins thought to be associated with sIBM, like $\beta$-amyloid, $\alpha$-synuclein, and tau protein, was present in the most of examined biopsies. All twelve patients were screened for the presence of causative mutations in TARDBP, VCP. HNRNPA1, and HNRNPA2B1 genes. Additionally, analysis of C9ORF72 hexanucleotide repeat expansion was performed. No causative mutations were found in any of the patients.

Conclusions: Our study provides the first - to our knowledge - comprehensive clinical, pathological, and genetic workup of a group of Polish patients.
\end{abstract}

Key words: inclusion body myositis, muscle biopsy, TDP-43, muscles' CT, genetic diagnostics. 


\section{Introduction}

Sporadic inclusion body myositis (SIBM) is an untreatable muscle disorder with weakness beginning after 50 years of age. It is more common in men than in women. Its pathogenesis remains undiscovered and the coexistence of inflammatory, degenerative, genetic, and environmental factors has been proposed. A possible genetic background is also considered. Its progressive course usually leads to immobilisation and severe dysphagia [3]. Both proximal and distal muscle weakness is observed, and the quadriceps, foot extensors, and deep finger flexors are most severely weakened. Creatine kinase (CK) activity may be slightly elevated. Electromyography studies (EMG) show myogenic or mixed myogenic and neurogenic pattern. Since clinical features of SIBM are not clearly delineated, morphological assessment of muscle biopsy is extremely important. Light microscopy examination reveals eosinophilic inclusions, necrotic fibres, and sometimes inflammatory infiltrates. Rimmed vacuoles are considered a hallmark of the disease. Additionally, immunohistochemistry shows deposits of various proteins such as $\beta$-amyloid, TDP-43 (TAR DNA-binding protein 43 ), tau protein, and $\alpha$-synuclein $[3,19]$. Electron microscopy (EM) examination reveals the presence of tubulofilamentous inclusions - both cytoplasmic and nuclear, degeneration of myofibrils, accumulation of abnormal mitochondria, and cytoplasmic bodies.

Inclusion body myositis diagnostic criteria were first proposed by Griggs et al. in 1995 [11]. In 2007 Needham and Mastaglia included additional muscle biopsy features, such as expression of MHC-I [13]. Later criteria were repeatedly modified, and the latest version comes from 2011 [14]. It is worth mentioning that among the major changes were the increase in the age of onset (to 45 years) and the introduction of three categories of IBM, namely "clinico-pathologically defined IBM", "clinically defined IBM", and "probable IBM". The authors emphasised that, due to sampling error or the early stage of the disease, not all pathological changes might be present in muscle biopsy [15]. Therefore, the diagnosis should be made according to both clinical and morphological features.

Over the last decades numerous pathogenic genes potentially involved in sporadic inclusion body myositis have been suggested. However, phenotype- genotype correlations are still poorly understood. Up to now all attempts to successfully treat inclusion body myositis failed. Neither corticosteroids and other immunosuppressive drugs nor intravenous immunoglobulins and plasma exchange were shown to improve clinical symptoms and prognosis.

Our study provides the first - to our knowledge - comprehensive clinical, pathological, and genetic workup of a group of eight sIBM Polish patients.

\section{Material and methods \\ Patients}

Clinical data of eight male patients aged 49 to 67 years with the diagnosis of sporadic IBM, hospitalised between 2002 and 2013 in the Department of Neurology of the Medical University of Warsaw were evaluated retrospectively. The course of the disease, distribution of muscle weakness, serum creatine kinase activity, and results of electrophysiological studies were evaluated. Additionally, four male patients aged 18 to 59 years with other primary muscle disorders were included in the study. The basis for including those patients was the presence of rimmed vacuoles in muscle biopsy. Among them there were three patients with clinically typical limb-girdle muscular dystrophy (LGMD) and one patient with genetically confirmed proximal myotonic myopathy (myotonic dystrophy type $2-\mathrm{DM} 2$ ). The "radiological control group" consisted of six men aged 58 to 65 years, with no signs and symptoms of neuromuscular disease. The study was approved by the Bioethical Committee at the Medical University of Warsaw.

\section{Computed tomography studies}

All computed tomography (CT) studies were performed using a 64-row scanner (Aquilion, Toshiba) without administration of contrast medium. The proximal one third of the thighs and lower legs were scanned. A low-dose protocol with automatic tube current modulation was used to minimise the radiation dose received by the patients.

Images in the axial (10 $\mathrm{mm}$ reconstructed slices) and coronal plane (5 $\mathrm{mm}$ reconstructed slices) were reviewed by an experienced musculoskeletal radiologist on the soft tissue (to grade muscles' atrophy) and bone window (to exclude bone pathology that may lead to muscle atrophy). Muscle atrophy was assessed on a five-point scale based on the scale 
originally developed by Goutallier et al. for grading muscular fatty degeneration in the rotator cuff (Table I) [10]. The control group consisted of six age-matched healthy volunteers (male, aged 58 to 65 years). The exclusion criteria for healthy volunteers included history of chronic lower back pain and significant trauma of the lower extremities.

\section{Muscle biopsy}

The open muscle biopsies were performed under local anaesthesia. The samples were obtained from the quadriceps in all eight patients with sIBM and in three patients with other myopathies. A biopsy from biceps brachii muscle was carried out in one patient. The muscle specimens were processed for further analyses using routine methods [6].

\section{Light microscopy}

All 12 muscle biopsies were assessed on light microscopy (LM). Biopsied tissue was frozen in isopentane cooled in liquid nitrogen, cut on a cryomicrotome with a slice thickness of $8 \mu \mathrm{m}$ and stained for a battery of routine histological and histochemical methods as well as for immunohistochemistry.

\section{Electron microscopy}

Ultrastructural examination was done in all patients. Samples for electron microscopy (EM) were fixed in glutaraldehyde and post-fixed in osmium tetroxide before embedding in Spurr embedding medium. Ultrathin sections of the selected areas were stained with uranyl acetate and counterstained with lead citrate. The samples were viewed with a JEM 1200 EX2 electron microscope.

\section{Immunohistochemical studies}

In six patients with inclusion body myositis the immunostaining for the $\beta$-amyloid (NCL-B-Amyloid antibody, Novocastra), TDP-43 (TARDBP monoclonal antibody, Abnova), tau protein (NCL-Tau-2 antibody, Novocastra), and $\alpha$-synuclein (Anti-Synuclein antibody, Chemicon) was performed using guidelines provided by each company. Additionally, the same procedures were applied in three of four patients with other myopathies. In two patients with SIBM and in one patient with LGMD the immunohistochemistry evaluation was not done according to the remote time of muscle biopsy.
Table I. Scale used for the grading of muscle atrophy in computed tomography (CT) (adapted from Goutallier et al., 1994) [10]

\begin{tabular}{|ll|}
\hline Atrophy grade & CT findings \\
\hline 0 & No atrophy \\
\hline 1 & $\begin{array}{l}\text { Minimal atrophy, muscle contains some } \\
\text { streaks of fat }\end{array}$ \\
\hline 2 & $\begin{array}{l}\text { Marked fatty degeneration with still more } \\
\text { muscle than fat }\end{array}$ \\
\hline 4 & $\begin{array}{l}\text { Advanced fatty degeneration with more fat } \\
\text { than muscle }\end{array}$ \\
& $\begin{array}{l}\text { Complete atrophy, muscle fibres replaced } \\
\text { by fat, only tendinous components pre- } \\
\text { served }\end{array}$ \\
\hline
\end{tabular}

\section{Genetic analyses}

All 12 patients were screened for the presence of known sIBM-causative mutations. Genomic DNA was isolated from peripheral blood leukocytes using standard methods. Intronic primers were used to amplify protein-coding exons of TARDBP and VCP, and exons coding for the prion-like domain of HNRNPA1 and HNRNPA2B1. Amplicons were purified with Exonuclease I/FastAP (Fermentas) and sequenced on an ABI PRISM 3130 Genetic Analyser (Applied Biosystems) using a BigDye Terminator v1.1 Cycle Sequencing Kit (Applied Biosystems). Primers for VCP and HNRNPA1, and HNRNPA2B1 were prepared as described by Hirano et al. 2015, and Seelen et al. 2014, respectively $[12,16]$. For TARDBP gene primers were designed using Primer 3 programme (Untergasser et al., 2012) [17]. Analysis of C9ORF72 hexanucleotide repeat was performed in two stages: fragment lengths analysis and the repeat-primed $P C R$ reaction previously described (DeJesus-Hernandez et al., 2011) [4].

\section{Results}

\section{Clinical characteristics}

Sporadic inclusion body myositis was diagnosed in eight male patients (number 1-8) with negative family history. Clinical characteristics are summarised in Table II. The age of onset varied from 46 to 65 years and the age of diagnosis from 49 to 67 years. The most often affected were the shoulder girdle (SG) and pelvic girdle (PG) muscles; however, in all patients distal upper limb (dUL) and distal lower limb (dLL) muscles involvement was also 
Table II. Patients' clinical characteristics

\begin{tabular}{|c|c|c|c|c|c|c|}
\hline Patient & Diagnosis & $\begin{array}{l}\text { Age of onset/ } \\
\text { Age of diagnosis }\end{array}$ & Affected muscles & CK (U/l) & NCS & EMG \\
\hline 1 & sIBM/c-p-def. & $59 / 63$ & SG, PG, dUL & 55 & $n$ & $\mathrm{M}+\mathrm{N}$ \\
\hline 2 & sIBM/ c-p-def. & $46 / 49$ & $\mathrm{SG}, \mathrm{PG}, \mathrm{dLL}$ & 107 & $n$ & $\mathrm{~N}$ \\
\hline 3 & sIBM/ c-p-def. & $65 / 67$ & SG, PG, dUL & 89 & $n$ & $n$ \\
\hline 4 & sIBM/ c-p-def. & $54 / 56$ & SG, PG, dUL, dLL & 237 & $n$ & M \\
\hline 5 & sIBM/c-def. & $49 / 60$ & $\mathrm{SG}, \mathrm{PG}, \mathrm{dLL}$ & 55 & $n$ & $n$ \\
\hline 6 & sIBM/prob. & $58 / 61$ & $\mathrm{dLL}$ & 19 & $n$ & $n$ \\
\hline 7 & sIBM/ c-p-def. & $54 / 60$ & $\mathrm{SG}, \mathrm{PG}, \mathrm{dUL}, \mathrm{dLL}$ & 110 & $n$ & $n$ \\
\hline 8 & sIBM/ c-p-def. & $55 / 55$ & SG, PG, dUL, dLL & 49 & $n$ & $M$ \\
\hline 9 & LGMD & $36 / 38$ & SG, PG & 96 & $n$ & M \\
\hline 10 & LGMD & $12 / 18$ & $\mathrm{SG}, \mathrm{PG}$ & 248 & $\mathrm{n}$ & M \\
\hline 11 & LGMD & $30 / 42$ & SG, PG & 527 & $n$ & $M$ \\
\hline 12 & DM2 & $49 / 59$ & $\mathrm{SG}, \mathrm{PG}, \mathrm{dUL}$ & 222 & A-m & $\mathrm{N}$ \\
\hline
\end{tabular}

SIBM - inclusion body myositis, c-p-def. - clinico-pathologically defined IBM, c-def. - clinically defined IBM, prob. - probable IBM, LGMD - limb-girdle muscular dystrophy, DM2 - myotonic dystrophy 2, SG - shoulder girdle muscles, PG - pelvic girdle muscles, dUL - distal muscles of upper limb, dLL - distal muscles of lower limb, CK - creatine kinase, NCS - nerve conduction studies, EMG - electromyography studies, $n$ - normal, A-m - axonal motor neuropathy, $M$ - myogenic, $N$-neurogenic, $M+N-$ myogenic and neurogenic

present. No calf muscle hypertrophy was observed. The course of the disease was progressive; nevertheless, all of the patients were mobile and only two of them used a walking stick. There were no reported difficulties in swallowing. Serum creatine kinase (CK) activity was moderately elevated, maximally up to seven times above the upper range (laboratory norm up to $34 \mathrm{U} / \mathrm{l})$. Electromyography disclosed a myogenic pattern from biceps brachii and vastus lateralis muscles in two patients, a neurogenic pattern from biceps brachii, vastus lateralis, interosseous I, and tibialis anterior muscles in one patient, and a myogenic pattern in the tibialis anterior muscle and neurogenic pattern in vastus lateralis muscle in one patient. In four cases electromyography was normal. Nerve conduction studies showed no abnormalities.

Additionally, four male patients (number 9-12) with other primary muscle disorders and with rimmed vacuoles on light microscopy examination were included. Three patients had clinically typical LGMD phenotype, without genetic confirmation of the subtype of LGMD. One patient had genetically confirmed proximal myotonic myopathy (myotonic dystrophy type 2 - DM2) (Table II). The age of onset ranged from 12 to 49 years and the age of diagnosis from 18 to 59 years in that group. The weakness of shoulder and pelvic girdle muscles was more pronounced than in sIBM patients. Calf muscles hypertrophy was present in all four patients. Serum creatine kinase activity was markedly elevated, maximally up to fifteen times above the upper range (laboratory norm up to $34 \mathrm{U} / \mathrm{l}$ ). Nerve conduction studies showed motor axonal neuropathy signs only in the patient with DM2. Electromyography from biceps brachii or vastus lateralis muscles presented a myogenic pattern in all patients with LGMD. In a patient with DM2 neurogenic pattern from biceps brachii and pseudomyotonic discharges in proximal muscles in the upper limb and in distal muscles in the lower limb were present.

\section{Computed tomography studies}

In the group of sIBM patients $\mathrm{CT}$ revealed a different degree of muscle atrophy in all but two patients. Muscle atrophy was symmetric and showed a slight predilection to the distal parts of muscle bellies in all but one patient. In the thighs, the atrophy was most pronounced within the quadriceps femoris and gracilis muscles, while in the lower legs it was within the medial head of the gastrocnemius muscle and within the tibialis anterior muscle (Fig. 1A-B). A summary of the degree of muscle atrophy results for the eight sIBM patients is presented in Fig. 2. 

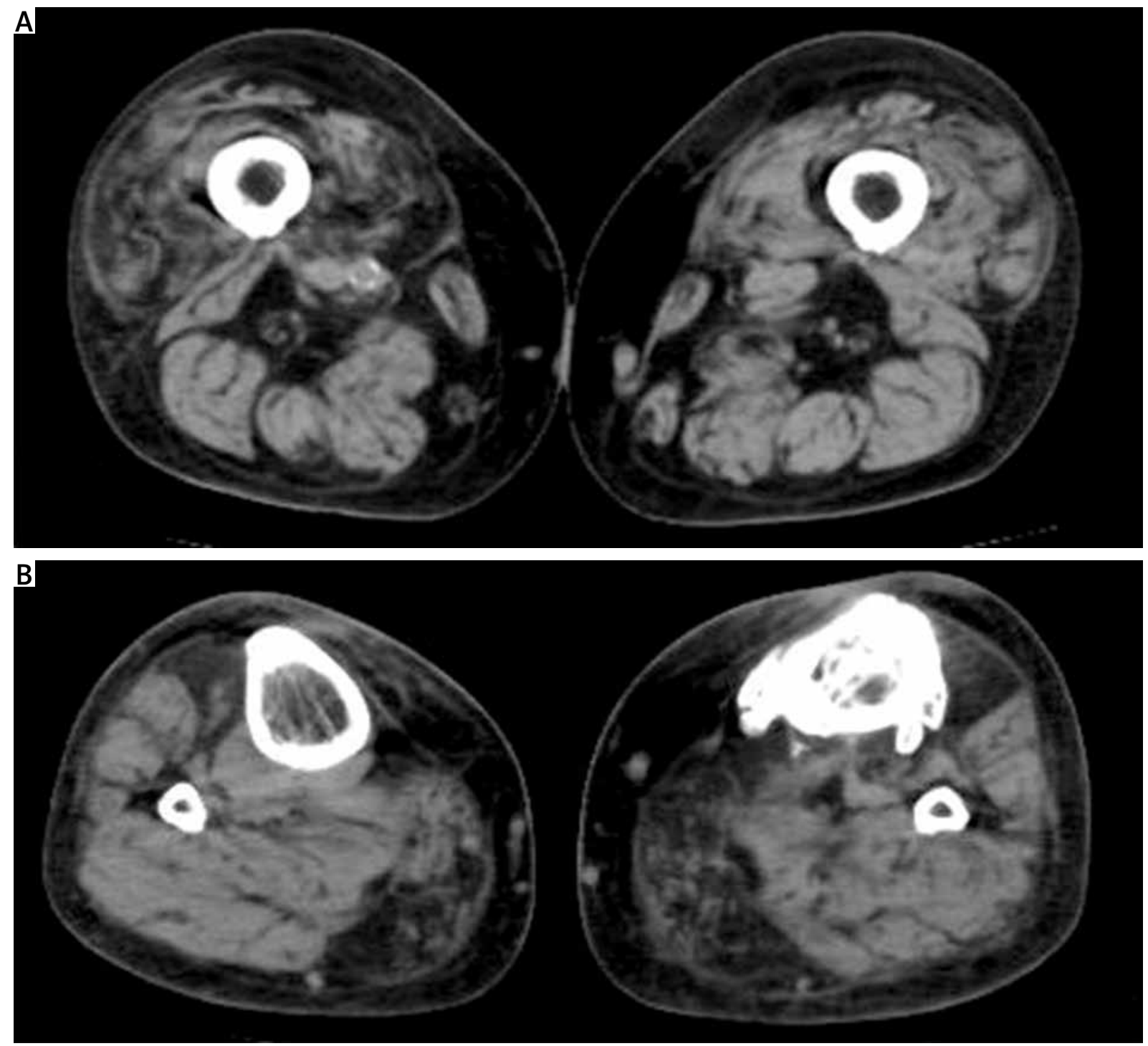

Fig. 1. Computed tomography images in a patient with "clinico-pathologically defined" sIBM: A) atrophy of quadriceps femoris muscle (grade 3 on the right, grade 2 on the left), B) atrophy of the medial head of the gastrocnemius muscle and within the tibialis anterior muscle (grade 4 on both sides).

In the control group, minimal muscle atrophy limited to the medial heads of the gastrocnemius muscle (grade 1 on the right, grade 2 on the left) was found in one 63-year-old man with no signs and symptoms of neuromuscular disease. All remaining participants of the control group had no muscle atrophy.

\section{Muscle biopsies assessment}

The results of light microscopic and ultrastructural evaluation are summarised in Table III and IV, respectively. The assessment of muscle samples on light microscopy revealed variation in fibre size in all 12 patients. There were many atrophic or hypertrophic fibres, fibres with internal nuclei, and muscle fibre splitting. Infiltration of mononuclear cells was observed in six patients with sIBM. "Rimmed vacuoles" considered characteristic for sIBM were noted in all 12 samples (Fig. 3A-D). Electron microscopy studies were performed in all patients (Fig. 4A-D). Myofibrillar disorganisation and mitochondrial abnormalities were observed in all biopsies. Lipofuscin accumulation was seen in all samples except for one patient with LGMD. Tubulofilamentous cytoplasmic inclusions composed of 15-18-nm tubulofil- 


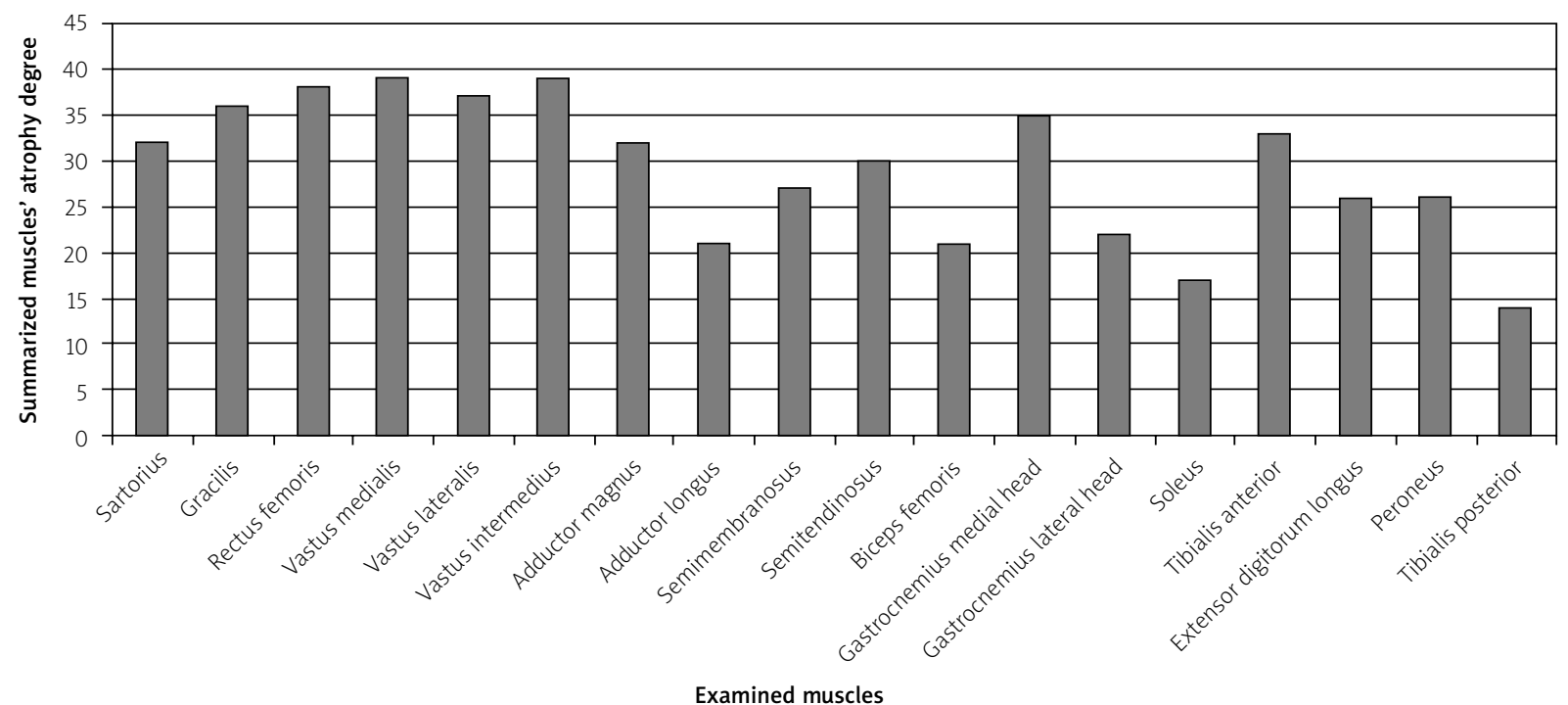

Fig. 2. Summarised muscle atrophy degree results.

aments were noted in two patients with sIBM. Both nuclear and cytoplasmic tubulofilamentous inclusions were observed in one sIBM and were present in one LGMD 18-year-old patient with biopsy and with other clinical and morphological findings supporting LGMD diagnosis.

Different patterns of accumulation of pathological proteins were observed in the examined biopsies. Immunohistochemical stains presented strong accumulation of TDP-43 in the subsarcolemmal and intracytoplasmic regions, in the atrophied fibres, in rimmed vacuoles, and in the blood vessels in half of the examined SIBM cases and in the patient with DM2 (Fig. 5A). $\beta$-amyloid aggregates, located in the subsarcolemmal and intracytoplasmic regions and in the atrophied fibres, were observed in all performed biopsies, being most prominent in the patient with DM2 (Fig. 5B). Tau protein accumulation, mostly located in the subsarcolemmal region, was present in six sIBM and in two control patients (Fig. 5C). Also

Table III. Light microscopy results

\begin{tabular}{|c|c|c|c|c|c|c|c|}
\hline Patient & $\begin{array}{l}\text { Varied fibre } \\
\text { size or shape }\end{array}$ & $\begin{array}{l}\text { Necrosis or } \\
\text { necrosis with } \\
\text { phagocytosis }\end{array}$ & $\begin{array}{l}\text { Mononuclear } \\
\text { cell invasion }\end{array}$ & $\begin{array}{l}\text { Rimmed } \\
\text { vacuoles }\end{array}$ & Inclusions & $\begin{array}{l}\text { Lobulated } \\
\text { fibres }\end{array}$ & Other \\
\hline 1 & + & + & + & + & + & + & $\begin{array}{c}\text { Connective tissue } \\
\text { hypertrophy }\end{array}$ \\
\hline 2 & + & + & + & + & + & - & - \\
\hline 3 & + & + & + & + & + & - & - \\
\hline 4 & + & + & + & + & + & - & - \\
\hline 5 & + & - & - & + & - & - & Ring fibres \\
\hline 6 & + & - & - & + & + & - & - \\
\hline 7 & + & + & + & + & + & - & - \\
\hline 8 & + & + & + & + & + & - & - \\
\hline 9 & + & + & + & + & + & + & - \\
\hline 10 & + & - & + & + & - & + & - \\
\hline 11 & + & + & + & + & + & - & - \\
\hline 12 & + & - & - & + & + & - & Internal nuclei \\
\hline
\end{tabular}

+ presence of abnormality, - absence of abnormality 
Table IV. Electron microscopy results

\begin{tabular}{|c|c|c|c|c|c|c|c|}
\hline Patient & $\begin{array}{l}\text { Cytoplasmic } \\
\text { inclusions }\end{array}$ & $\begin{array}{c}\text { Nuclear } \\
\text { inclusions }\end{array}$ & $\begin{array}{c}\text { Myofibril } \\
\text { abnormalities }\end{array}$ & $\begin{array}{l}\text { Mitochondrial } \\
\text { abnormalities }\end{array}$ & $\begin{array}{l}\text { Cytoplasmic } \\
\text { bodies }\end{array}$ & $\begin{array}{l}\text { Lipofuscin } \\
\text { accumulation }\end{array}$ & $\begin{array}{c}\text { Myelin } \\
\text { structures }\end{array}$ \\
\hline 1 & + & - & + & + & - & + & + \\
\hline 2 & - & - & + & + & + & + & - \\
\hline 3 & - & - & + & + & - & + & - \\
\hline 4 & + & + & + & + & + & + & + \\
\hline 5 & - & - & + & + & - & + & - \\
\hline 6 & - & - & + & + & - & + & - \\
\hline 7 & + & - & + & + & - & + & + \\
\hline 8 & - & - & + & + & + & + & + \\
\hline 9 & - & - & + & + & - & - & - \\
\hline 10 & + & + & + & + & + & + & + \\
\hline 11 & - & - & + & + & - & + & - \\
\hline 12 & - & - & + & + & - & + & - \\
\hline
\end{tabular}

+ presence of abnormality, - absence of abnormality
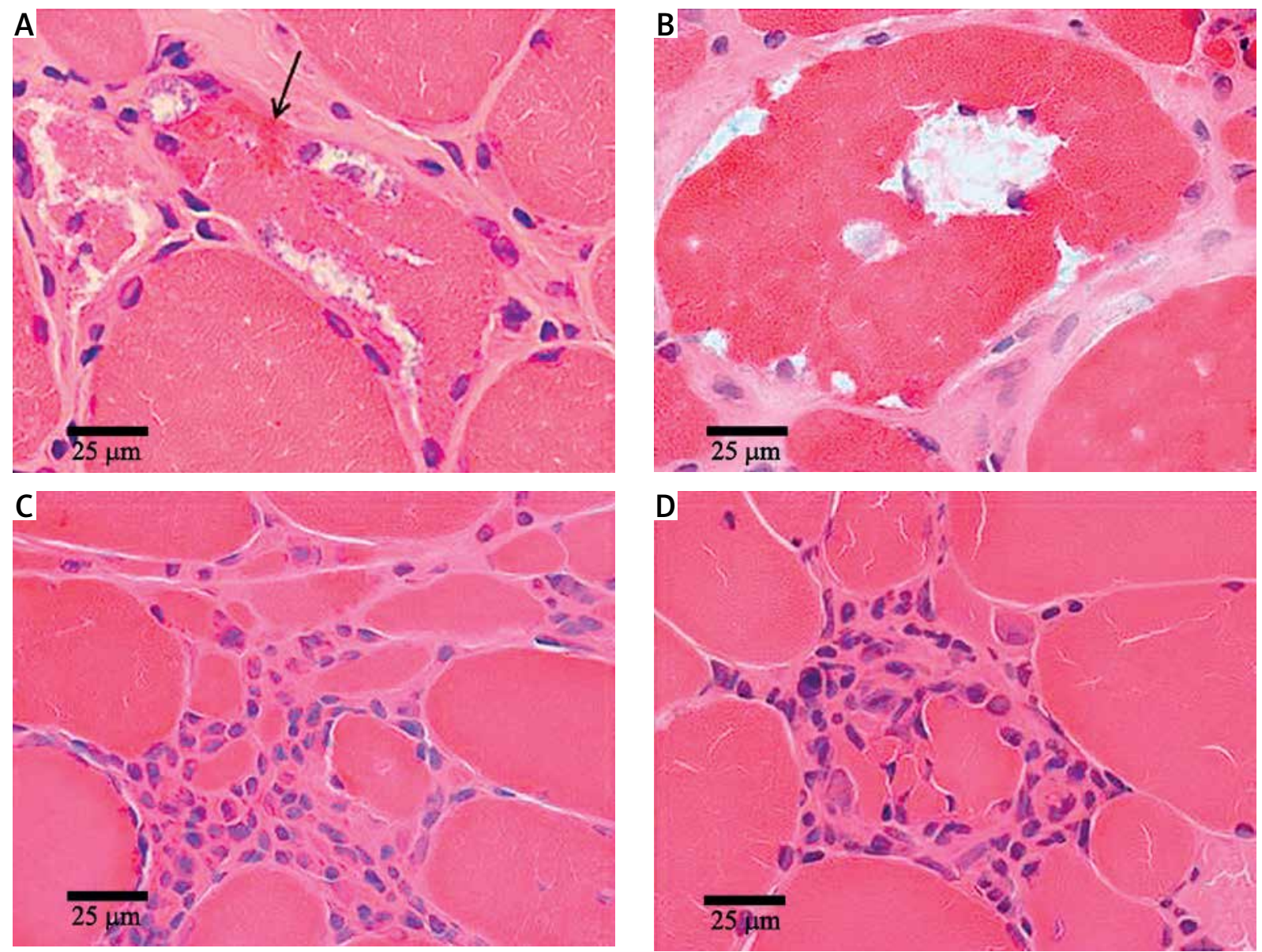

Fig. 3. Haematoxylin-eosin staining from sIBM patients: A) cytoplasmic inclusions (arrow), B) rimmed vacuoles, C) mononuclear cells invasion, D) necrosis with phagocytosis. Bar $25 \mu \mathrm{m}$. 

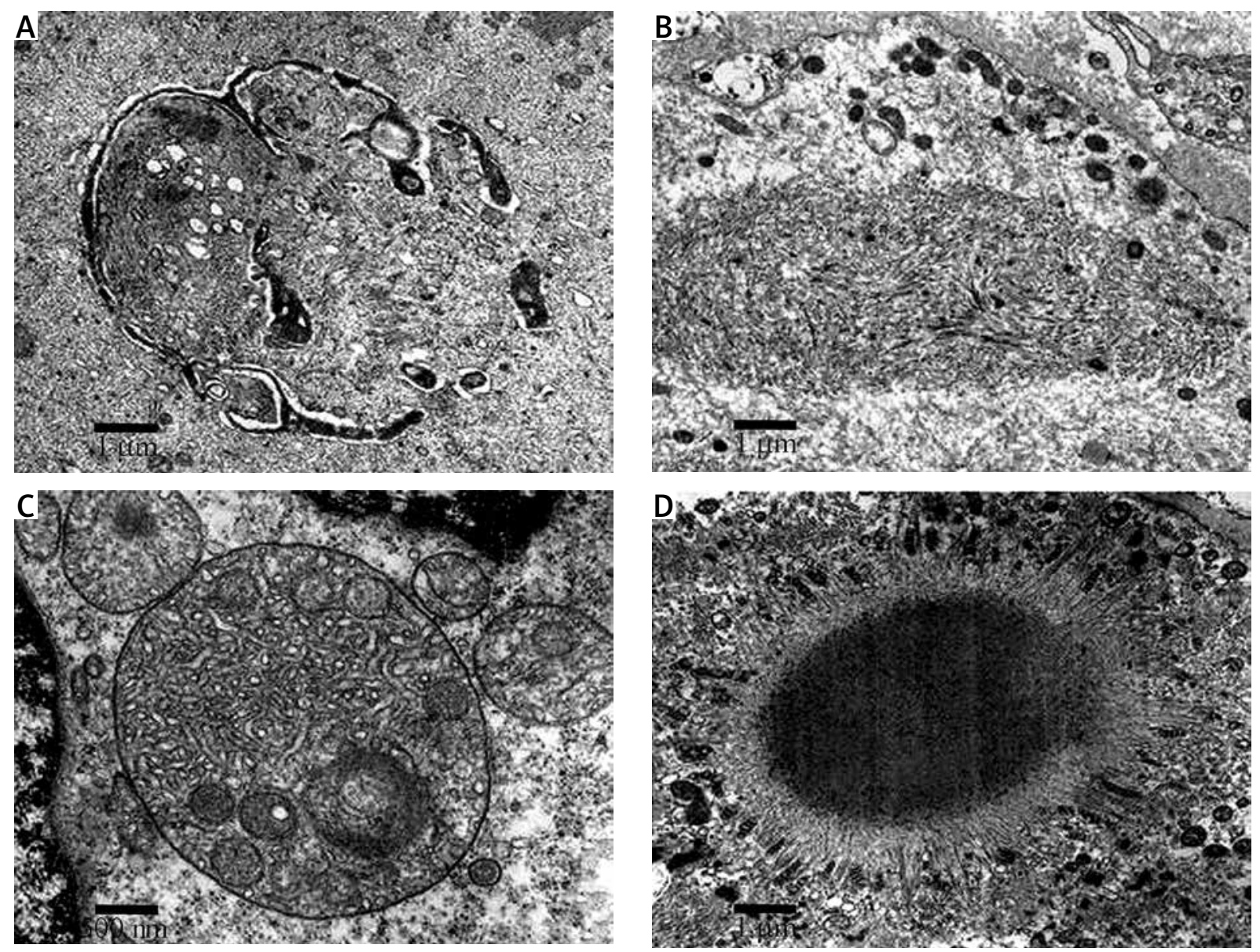

Fig. 4. Electron microscopy results from sIBM patients: A) nuclear inclusions, B) cytoplasmic inclusions,

C) mitochondrial abnormalities, D) cytoplasmic body. Bar $1 \mu \mathrm{m}$ for A), B), and D); bar $500 \mathrm{~nm}$ for C).

$\alpha$-synuclein aggregates, located in the subsarcolemmal and intracytoplasmic regions mostly in the atrophied fibres, were noted in all examined cases (Fig. 5D). The results of immunohistochemical studies are listed in Table V.

Based on such comprehensive evaluation, according to Rose et al.'s criteria, six patients (numbers 1-4, 7, and 8) were classified as "clinico-pathologically defined IBM", one patient (number 5) as "clinically defined IBM", and one patient (number 6) as "probable IBM" [14].

\section{Genetic analyses}

No causative mutations were found in the protein-coding sequences of TARDBP, VCP, HNRNPA1, HNRNPA2B1, and C9ORF72 genes in any of the examined patients with sIBM and other myopathies. Two novel and probably not pathogenic intronic insertions and known exonic and intronic polymorphisms were identified in VCP and HNRNPA2B1 genes (see Table VI).

\section{Discussion}

The pathogenic mechanisms of SIBM are still unknown. It is believed that both environmental and genetic factors can contribute to the disease process. Clinical diagnosis of SIBM presents many problems and in most cases requires additional morphological, radiological, and genetic assessment. No single procedure is unreservedly precise, and only occasionally can the disease be confirmed by specific mutation. The primary diagnostic criteria for sporadic IBM were first proposed by Griggs et al. in 1995 and they emphasised the role of skeletal muscle biopsy [11]. Inclusion criteria covered duration of the disease above six months, age of onset above 30 years, both proximal and distal muscle weakness of upper and lower limbs, and negative family history. 

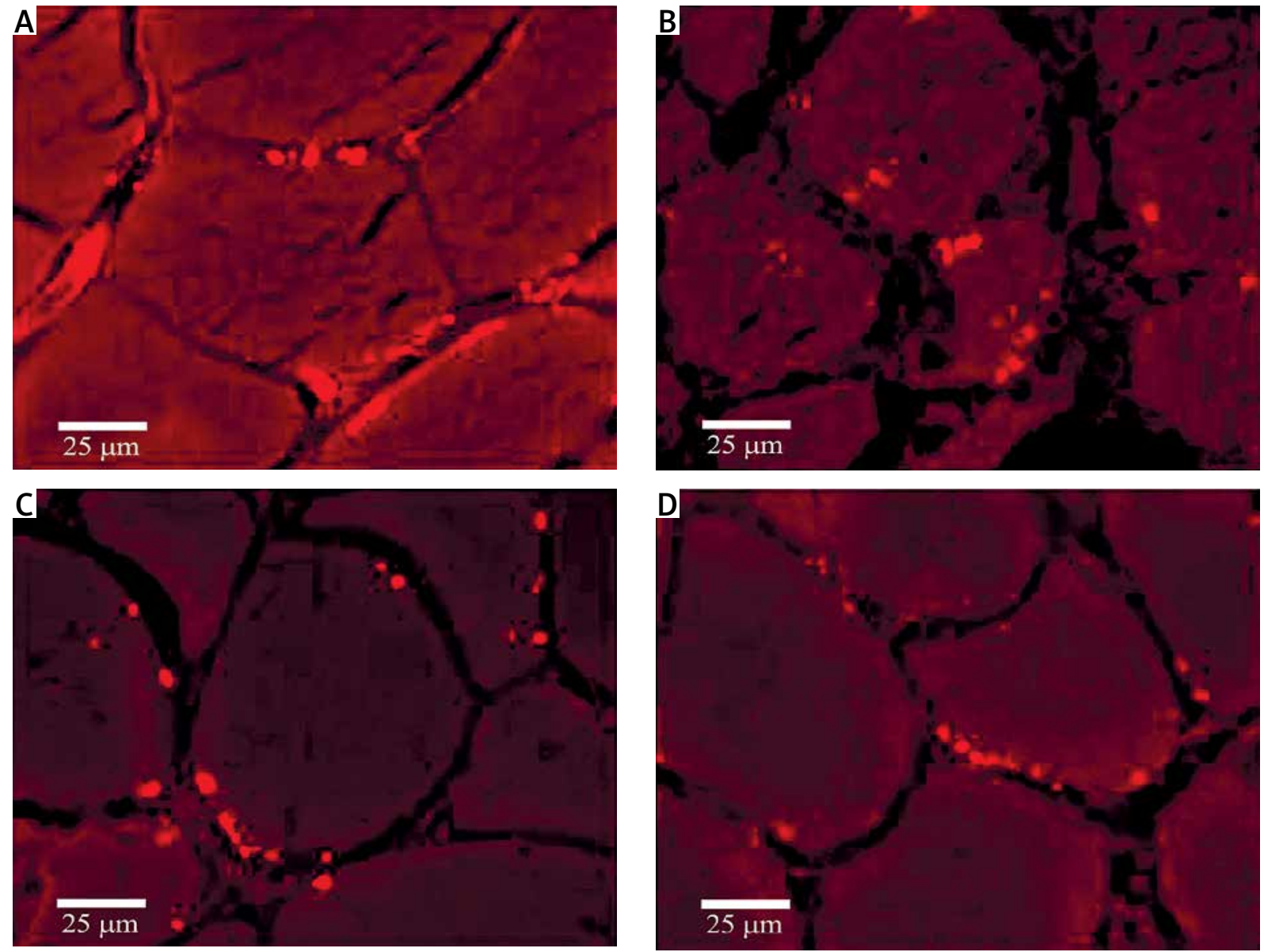

Fig. 5. Immunohistochemical results from sIBM patients: A) TDP-43 aggregates, B) $\beta$-amyloid aggregates, C) tau protein aggregates, D) $\alpha$-synuclein aggregates. Bar $25 \mu \mathrm{m}$.

Table V. Immunohistochemistry results

\begin{tabular}{|c|c|c|c|c|}
\hline Patient & TDP-43 & $\beta$-amyloid & tau protein & $\alpha$-synuclein \\
\hline 1 & $++; S S, A F, I C$ & + +; SS, AF & +; SS, AF & +; SS, AF, IC \\
\hline 2 & ND & ND & ND & ND \\
\hline 3 & $+; \mathrm{SS}, \mathrm{AF}, \mathrm{V}$ & $+; \mathrm{SS}, \mathrm{AF}$ & $+; \mathrm{SS}, \mathrm{AF}$ & $+; \mathrm{SS}, \mathrm{AF}$ \\
\hline 4 & ND & ND & ND & ND \\
\hline 5 & $++; S S, I C$ & $++; S S$ & +++ ;SS & ND \\
\hline 6 & ++ ; SS & + +;SS & $++; S S$ & + ; SS \\
\hline 7 & $+; \mathrm{SS}, \mathrm{AF}, \mathrm{V}$ & $++; S S, A F, I C$ & $+; \mathrm{AF}$ & $+; \mathrm{SS}, \mathrm{AF}$ \\
\hline 8 & $++; A F, R V, V$ & + +; SS & + ; SS, AF & $+; S S, A F, I C$ \\
\hline 9 & + +;SS & + ; SS & ND & ND \\
\hline 10 & ND & ND & ND & ND \\
\hline 11 & $+; \mathrm{SS}, \mathrm{AF}, \mathrm{V}$ & $+; A F, I C$ & + +;SS & $+; \mathrm{SS}, \mathrm{AF}$ \\
\hline 12 & ++ ; SS, V & $+++; S S, A F, I C$ & $+++; S S, A F, I C, V$ & $+; \mathrm{SS}, \mathrm{AF}$ \\
\hline
\end{tabular}

+ slight accumulation, ++ strong accumulation, +++ excessive accumulation

$S S$ - subsarcolemmal, AF - atrophied fibres, IC - intracytoplasmic, $V$-blood vessels, $R V$-rimmed vacuoles ND - not done 
Table VI. Genetic analyses results

\begin{tabular}{|lcccc|}
\hline Gene & dbSNPs & Localization & Most severe potential consequence & MAF \\
\hline TARDBP & rs968545 & Intron & Unknown & 0.125 (T) \\
\hline VCP & rs4133584 & Intron & Unknown & 0.458 (C) \\
\hline & rs10972300 & Intron & Unknown & 0.208 (A) \\
\hline & rs514492 & Intron & Splice region variant & 0.167 (G) \\
\hline & rs2258240 & Intron & Unknown & 0.292 (INS) \\
\hline rs562381 & Intron & Unknown & 0.500 (G) \\
\hline HNRNPA1 & rs684562 & Exon & Non-coding transcript exon variant & 0.333 (T) \\
\hline HNRNPA2B1 & g.12606_12607insGAAGATAA* & Intron & Splice region variant & 0.125 (A) \\
\hline
\end{tabular}

MAF-minor allele frequency

*Novel variant, VCP NCBI Reference Sequence: NG_007887.1, HNRNPA2B1 NCBI Reference Sequence: NG_029680.1

Laboratory abnormalities included serum creatine kinase activity not more than 12-times normal, typical muscle biopsy changes, and diversified pattern of electromyography. "Definite" inclusion body myositis was recognised when muscle biopsy presented mononuclear cell invasion of non-necrotic muscle fibres, vacuolated muscle fibres and intracellular amyloid deposits, or 15-18 nm tubulofilaments. In such cases no additional clinical and laboratory criteria were necessary. "Possible" inclusion body myositis was considered when muscle biopsy presented only inflammatory features and the patient exhibited all of the clinical and laboratory symptoms mentioned above. Later Needham and Mastaglia applied different morphological criteria [13]; however, they emphasised that the lack of particular changes in muscle biopsy cannot exclude IBM. They introduced the term "probable" inclusion body myositis to characterise patients with incomplete biopsy criteria, and the term "possible" IBM to distinguish patients with atypical muscle involvement and non-specific muscle biopsy findings. Finally, Rose in cooperation with the European Neuromuscular Centre (ENMC) developed the so-called "IBM Research Diagnostic Criteria 2011 ", published as a workshop report in 2013 [14]. The symptoms had to last for at least 12 months, the age of onset was increased to 45 years, and serum creatine kinase activity had to be no greater than 15-times above the upper limit of normal. The knee extension and finger flexion weakness had to be more pronounced than hip flexion weakness and shoulder abduction weakness, respectively. If these features were accompanied by all pathological changes (which covered endomysial inflammatory infiltrates, rimmed vacuoles, $\beta$-amyloid or TDP-43 accumulation, or inclusions composed of 15-18 nm tubulofilaments in EM), then "clinico-pathologically defined IBM" could be recognised. When not all pathological changes were present, the diagnosis was classified as "clinically defined IBM". And when not all clinical features were expressed, "probable IBM" was diagnosed. Although patients included in our study were hospitalised between 2002 and 2013 in the Department of Neurology, their clinical and morphological data were re-evaluated according to Rose et al.'s criteria developed in 2011. Application of those criteria in our study allowed us to recognise "clinico-pathologically defined IBM" in six cases, "clinically defined IBM" in one case, and "probable IBM" in one.

According to the literature, the muscle assessment on light microscopy should be focused on vacuolar degeneration and atrophy of muscle fibres with mononuclear cell infiltrations. The so-called "rimmed vacuoles" are considered the hallmark for SIBM; nevertheless, they are seen in many other muscle disorders, for example in inclusion body myopathy with Paget's disease and frontotemporal dementia (IBMPFD), in oculopharyngeal muscular dystrophy, or in myofibrillar myopathies [1]. Additionally, tubulofilamentous inclusions in the cytoplasm or in the nuclei can be found on electron microscopy in a variety of neuromuscular diseases $[7,8]$. Numerous studies have showed deposits of 
different proteins in SIBM and none was disease specific [19]. The presence of TDP-43 aggregates in SIBM was highlighted in several articles [5]. In healthy subjects TDP-43 is present mostly in the nucleus, whereas in SIBM cases it is seen as non-nuclear sarcoplasmic deposits [15]. However, the precise role of TDP-43 accumulation is still unknown; it may cause neuronal dysfunction and disturb transcriptional regulation and RNA processing. We detected no TARDBP mutation associated with IBM, which is consistent with Qian Gang et al.'s study (Qian Gang et al., 2014) [9]. We did not detect pathogenic hexanucleotide expansion in C9ORF72, which is the most common cause of amyotrophic lateral sclerosis (ALS) and frontotemporal dementia (FTD) with TDP-43 inclusions (Weihl et al., 2015) [18]. Other possible genes associated with the disease may be HNRNPA1 and HNRNPA2B1 [16].

Mutations in prion-like domain of the RNA binding genes heterogeneous nuclear ribonucleoproteins $A 1$ and $A 2 B 1$ were shown to cause multi-system proteinopathy (MSP), which may be clinically presented as IBM, FTD, ALS, or Paget's disease (Seelen et al., 2014) [16]. However, our results, as well as the analysis of 31 patients from the Dutch population have shown no causative mutation in clinically defined IBM [16]. Our study also reveals no mutation in $V C P$, known to be causative for IBM.

The presented study describes eight patients with sporadic inclusion body myositis and four with other primary muscle disorders. This is the first, to our knowledge, such comprehensive workup of Polish patients with sIBM. Clinical findings were similar to those described in the literature and correlated with criteria proposed by Rose et al. [14]. The muscle involvement highlighted on computed tomography studies was comparable to that known from previous reports [2].

Morphological and immunohistochemical assessments proved that none of the changes are disease specific. The presence of TDP-43 and other protein aggregates were seen in all examined SIBM patients as well as in patients with other myopathies. It seems that, regardless of the primary cause, muscle fibre degeneration may be connected with the formation of various protein aggregates [14].

\section{Conclusions}

Our analysis of eight SIBM patients and four patients with other myopathies did not indicate the role of TDP-43 in SIBM pathogenesis. Muscle biopsy, although in some cases not conclusive for sIBM diagnosis, remains important for excluding other possible diagnoses of neuromuscular disorders. The analysis of known genes involved in SIBM pathogenesis did not confirm the genetic background in the examined group of eight Polish patients.

\section{Disclosure}

Authors report no conflict of interest.

\section{Acknowledgments}

This work has been supported by grant N N402 434238 from the Ministry of Science and Higher Education.

Dr Jo Lewkowicz edited our paper from the language point of view.

\section{References}

1. Cai H, Yabe I, Sato K, Kano T, Nakamura M, Hozen H, Sasaki H. Clinical, pathological, and genetic mutation analysis of sporadic inclusion body myositis in Japanese people. J Neurol 2012; 259: 1913-1922.

2. Cox FM, Reijnierse M, van Rijswijk CSP, Wintzen AR, Verschuuren J, Badrising UA. Magnetic resonance imaging of skeletal muscles in sporadic inclusion body myositis. Rheumatology 2011; 50: 1153-1161.

3. Dalakas MC. Sporadic inclusion body myositis - diagnosis, pathogenesis and therapeutic strategies. Nat Clin Pract Neurol 2006; 2: 437-447.

4. DeJesus-Hernandez M, Mackenzie IR, Boeve BF, Boxer AL, Baker M, Rutherford NJ, Nicholson AM, Finch NA, Flynn H, Adamson J, Kouri N, Wojtas A, Sengdy P, Hsiung GY, Karydas A, Seeley WW, Josephs KA, Coppola G, Geschwind DH, Wszolek ZK, Feldman H, Knopman DS, Petersen RC, Miller BL, Dickson DW, Boylan KB, Graff-Radford NR, Rademakers R. Expanded GGGGCC hexanucleotide repeat in noncoding region of C9ORF72 causes chromosome 9p-linked FTD and ALS. Neuron 2011; 72 : 245-256.

5. Dubourg O, Wanschitz J, Maisonobe T, Béhin A, Allenbach Y, Herson S, Benveniste O. Diagnostic value of markers of muscle degeneration in sporadic inclusion body myositis. Acta Myol 2011; 30: 103-108.

6. Dubowitz V, Sewry CA. The procedure of muscle biopsy. Histological and histochemical stains and reactions. In: Muscle biopsy: A Practical Approach. 3rd ed. Saunders Elsevier, Philadelphia 2007; pp. 3-40.

7. Fidziańska A, Kamińska A, Ryniewicz B. Congenital myopathy with tubular aggregates and tubulofilamentous IBM-type inclusions. Neuropediatrics 2005; 36: 35-39.

8. Fidzianska A, Ryniewicz B, Shen XM, Engel AG. IBM-type inclusions in a patient with slow-channel syndrome caused by 
a mutation in the AChR epsilon subunit. Neuromuscul Disord 2005; 15: 753-759.

9. Gang Q, Bettencourt C, Machado P, Hanna MG, Houlden H. Spo radic inclusion body myositis: the genetic contributions to the pathogenesis. Orphanet J Rare Dis 2014; 9: 88.

10. Goutallier D, Postel JM, Bernageau J, Lavau L, Voisin MC. Fatty muscle degeneration in cuff ruptures. Pre- and postoperative evaluation by CT scan. Clin Orthop Relat Res 1994; 304: 78-83.

11. Griggs RC, Askanas V, DiMauro S, Engel A, Karpati G, Mendell JR, Rowland LP. Inclusion body myositis and myopathies. Ann Neurol 1995; 38: 705-713.

12. Hirano M, Nakamura Y, Saigoh K, Sakamoto H, Ueno S, Isono C, Mitsui Y, Kusunoki S. VCP gene analyses in Japanese patients with sporadic amyotrophic lateral sclerosis identify a new mutation. Neurobiol Aging 2015; 36: 1604.e1-6.

13. Needham M, Mastaglia FL. Inclusion body myositis: current pathogenic concepts and diagnostic and therapeutic approaches. Lancet Neurol 2007; 6: 620-631.

14. Rose MR, ENMC IBM Working Group. 188th ENMC International Workshop: Inclusion Body Myositis, 2-4 December 2011, Naarden, The Netherlands. Neuromuscul Disord 2013; 23: 1044-1055.

15. Salajegheh M, Pinkus JL, Taylor JP, Amato AA, Remedios N, Baloh RH, Greenberg SA. Sarcoplasmic redistribution of nuclear TDP-43 in inclusion body myositis. Muscle Nerve 2009; 40: 19-31.

16. Seelen M, Visser AE, Overste DJ, Kim HJ, Palud A, Wong TH, van Swieten JC, Scheltens P, Voermans NC, Baas F, de Jong JM, van der Kooi AJ, de Visser M, Veldink JH, Taylor JP, Van Es MA, van den Berg LH. No mutations in hnRNPA1 and hnRNPA2B1 in Dutch patients with amyotrophic lateral sclerosis, frontotemporal dementia, and inclusion body myopathy. Neurobiol Aging 2014; 35: 1956.e9-1956.e11.

17. Untergasser A, Cutcutache I, Koressaar T, Ye J, Faircloth BC, Remm M, Rozen SG. Primer3 - new capabilities and interfaces. Nucleic Acids Res 2012; 40: e115.

18. Weihl CC, Baloh RH, Lee Y, Chou TF, Pittman SK, Lopate G, Allred $P$, Jockel-Balsarotti J, Pestronk A, Harms MB. Targeted sequencing and identification of genetic variants in sporadic inclusion body myositis. Neuromuscul Disord 2015; 25: 289-296.

19. Weihl CC, Pestronk A. Sporadic Inclusion Body Myositis: Possible pathogenesis inferred from biomarkers. Curr Opin Neurol 2010; 23: 482-488. 